\title{
Risk factors for breast cancer in a population with high incidence
} rates

\author{
Margaret Wrensch ${ }^{1}$, Terri Chew ${ }^{1}$, Georgianna Farren², Janice Barlow ${ }^{2}$, Flavia Belli², \\ Christina Clarke ${ }^{3}$, Christine A Erdmann ${ }^{4}$, Marion Lee ${ }^{1}$, Michelle Moghadassi ${ }^{1}$, \\ Roni Peskin-Mentzer², Charles P Quesenberry Jr5, Virginia Souders-Mason², Linda Spence ${ }^{2}$, \\ Marisa Suzuki ${ }^{1}$ and Mary Gould ${ }^{2+}$ \\ 1 University of California, San Francisco, California, USA \\ 2Marin Breast Cancer Watch, San Rafael, California, USA \\ ${ }^{3}$ Northern California Cancer Center, Union City, California, USA \\ ${ }^{4}$ Lawrence Berkeley National Laboratory, Berkeley, California, USA \\ ${ }^{5}$ Kaiser Foundation Research Institute, Oakland, California, USA \\ ${ }^{+}$Deceased \\ Corresponding author: Margaret Wrensch (e-mail: wrensch@itsa.ucsf.edu)
}

Received: 19 Dec 2002 Revisions requested: 5 Mar 2003 Revisions received: 21 Mar 2003 Accepted: 7 Apr 2003 Published: 29 Apr 2003

Breast Cancer Res 2003, 5:R88-R102 (DOI 10.1186/bcr605)

(c) 2003 Wrensch et al., licensee BioMed Central Ltd (Print ISSN 1465-5411; Online ISSN 1465-542X). This is an Open Access article: verbatim copying and redistribution of this article are permitted in all media for any purpose, provided this notice is preserved along with the article's original URL.

See related Commentary: http://breast-cancer-research.com/content/5/5/232

\begin{abstract}
Background: This report examines generally recognized breast cancer risk factors and years of residence in Marin County, California, an area with high breast cancer incidence and mortality rates.

Methods: Eligible women who were residents of Marin County diagnosed with breast cancer in 1997-99 and women without breast cancer obtained through random digit dialing, frequencymatched by cases' age at diagnosis and ethnicity, participated in either full in-person or abbreviated telephone interviews.

Results: In multivariate analyses, 285 cases were statistically significantly more likely than 286 controls to report being premenopausal, never to have used birth control pills, a lower highest lifetime body mass index, four or more mammograms in 1990-94, beginning drinking after the age of 21 , on average

drinking two or more drinks per day, the highest quartile of pack-years of cigarette smoking and having been raised in an organized religion. Cases and controls did not significantly differ with regard to having a first-degree relative with breast cancer, a history of benign breast biopsy, previous radiation treatment, age at menarche, parity, use of hormone replacement therapy, age of first living in Marin County, or total years lived in Marin County. Results for several factors differed for women aged under 50 years or 50 years and over.

Conclusions: Despite similar distributions of several known breast cancer risk factors, case-control differences in alcohol consumption suggest that risk in this high-risk population might be modifiable. Intensive study of this or other areas of similarly high incidence might reveal other important risk factors proximate to diagnosis.
\end{abstract}

Keywords: breast neoplasms, case-control study, epidemiology, risk factors

\section{Introduction}

The San Francisco Bay Area reports some of the highest breast cancer rates in the world [1,2]. Within this region, appropriately adjusted breast cancer incidence and mortality rates are highest in Marin County, a small, urban county of 250,000 predominantly white, non-Hispanic res- idents located immediately north of the city of San Francisco. Averaged over the period 1995-99, age-adjusted invasive breast cancer rates per 100,000 white, non-Hispanic women were 199 in Marin County, 155 in the rest of the San Francisco Bay Area, and 144 in the United States as a whole [2]. Pronounced international and national 
geographic variation in breast cancer rates has been studied extensively [3-8] and has been attributed variously to population differences in the use of mammographic screening, diet, physical activity, body size, alcohol consumption, and socioeconomic and reproductive factors $[5-7,9,10]$. However, studies of breast cancer incidence among Asian migrants to North America have noted associations with age at migration, suggesting additional important influences of childhood or adolescent exposures or experiences [4-6]. With regard to Marin County, an earlier study by the Northern California Cancer Center (NCCC) using census data suggested that the high incidence was associated with the sociodemographic characteristics of the county and not with a geographically specific exposure [10] and that women living in Marin County have a higher prevalence of some recognized breast cancer risk factors, such as high education and income as well as low and late parity $[10,11]$.

In 1997, members of Marin Breast Cancer Watch, a grassroots non-profit organization of breast cancer survivors and advocates, recruited researchers to help them to investigate causes of breast cancer in Marin County more thoroughly; these community members actively participated in all aspects of conducting this study.

In this population-based case-control study of women residing in Marin County, we asked about generally recognized risk factors $[5,12]$ and a variety of adolescent physical, psychological, and social factors. The main purpose in this first report is to compare generally recognized breast cancer risk factors, as well as childhood and adolescent socioeconomic factors and years of residence in Marin County for women with and without breast cancer. Although it was not the purpose of this study to explain the high rates of breast cancer in Marin County, one hypothesis of the study was that if exposures specific to Marin County were to some degree responsible for the high rates there, one would expect women with breast cancer to have lived longer in Marin County than control women after adjustment for generally recognized breast cancer risk factors. The thought was that finding such a difference would provide justification for a more detailed exploration of exposures specific to Marin County, whereas no difference would motivate research into other explanations. Thus, this initial report provides important information for planning future studies in this and other high-risk areas.

\section{Methods}

\section{Case-control ascertainment}

Eligible cases included any female resident of Marin County with a diagnosis of primary breast cancer between July 1997 and June 1999 if under 50 years of age, and between July 1997 and March 1999 if 50 years old or older at diagnosis (Table 1). Dates were chosen to yield about 300 women overall, 100 under 50 years of age and
Table 1

Participation rates, age and ethnicity of women with breas cancer and controls, Marin County, California, 1997-99

\begin{tabular}{|c|c|c|c|c|}
\hline \multirow[b]{2}{*}{ Variable } & \multicolumn{2}{|c|}{ Controls } & \multicolumn{2}{|c|}{ Cases } \\
\hline & $N$ & $\%$ & $N$ & $\%$ \\
\hline Eligiblesa & 328 & & 401 & \\
\hline \multicolumn{5}{|l|}{ Study participants ${ }^{b}$} \\
\hline Full interview & 305 & 93 & 300 & 75 \\
\hline Short interview & 16 & 5 & 36 & 9 \\
\hline Deceased & 0 & 0 & 15 & 4 \\
\hline Refusal/other & 7 & 2 & 50 & 12 \\
\hline Total & 328 & & 401 & \\
\hline \multicolumn{5}{|l|}{ Ethnicity } \\
\hline White & & 98 & & 94 \\
\hline Full interview & 300 & & 285 & \\
\hline Short interview & 16 & & 30 & \\
\hline Non-white & & 2 & & 6 \\
\hline Full interview & 5 & & 15 & \\
\hline Short interview & 0 & & 6 & \\
\hline Total & 321 & & 336 & \\
\hline Age & Mean \pm SEM & Median & Mean \pm SEM & Median \\
\hline Full interview & $55.1 \pm 0.36$ & 55 & $55.4 \pm 0.55$ & 55 \\
\hline Short interview & $55.8 \pm 2.83$ & 55 & $56.7 \pm 1.93$ & 58 \\
\hline All women & $55.2 \pm 0.54$ & 55 & $55.6 \pm 0.53$ & 55 \\
\hline
\end{tabular}

aCases eligibility: less than 50 years old, newly diagnosed with primary breast cancer between 1 July 1997 and 30 June 1999 or at least 50 years old and diagnosed between 1 July 1997 and 22 March 1999. For controls, 3945 random digit numbers were called: 1640 (41\%) either were not in service, were a business, fax or modem number, or were outside Marin County; 920 (23\%) were reached but no resident was eligible; 289 (7\%) yielded refusal with no additional information; $733(19 \%)$ were in service but were either busy or did not answer (of which 394 were closed out after 10 call attempts and 339 had fewer than 10 call attempts made and were not closed out by the time that control recruitment ended); and 347 (9\%) yielded an eligible subject. Of 347 women identified as eligible, 328 were contacted.

bFive control women refused any participation and two could not be contacted. Thirty-six cases refused any participation, physicians refused contact for three cases, two cases could not be interviewed in English, we were unable to contact six cases, and three cases were mistakenly coded as ineligible.

200 aged 50 and over. The sample size was planned with $80 \%$ statistical power to detect 2.1 -fold odds ratios (ORs) between the highest and lowest quartiles of any continuous variable, 1.9-fold ORs for quartile trend, a 0.25-year case-control difference in age at menarche, and a 3.5-year case-control difference in years lived in Marin. Names and addresses of these women and their physicians were obtained from the cancer registry operated by the NCCC, a participant in the National Cancer Institute's (NCl's) 
Surveillance, Epidemiology, and End Results program. After receiving an introductory letter, women who did not refuse by postcard were telephoned by a study interviewer to arrange a time and place for the in-person interview.

Control women without breast cancer were ascertained through random digit dialing (RDD) by using a modification of the method of Waksberg $[13,14]$ and were frequency-matched to cases by age at diagnosis (within 5 years) and ethnicity. To maximize the chances of reaching residential numbers, seed numbers used for dialing included the area code plus the first five digits of the study's cases and all cancer cases diagnosed in Marin County in 1998. All possible numbers other than the cases' numbers were generated and randomly sorted. The RDD contractor also generated a batch of about 300 random numbers from marketing sources to increase the number of calls to households with women aged 65 years and older. Randomly sorted numbers were called sequentially. Two survey research companies (Survey Methods, and Field Research Corporation, both of San Francisco, California) conducted RDD and preliminary eligibility screening of controls. RDD was conducted from April 2000 to August 2001.

The University of California, San Francisco, Committee on Human Research approved the study methods, and informed consent was obtained from all participants.

\section{Interviews}

The approximately 2-hour in-person interviews were conducted at a place of each subject's choosing. Because many questions required the recall of adolescent factors, the interview used three memory tools developed during the pilot phase of this study: a visualization exercise, a magnetic memory board, and photographs of girls at various stages of development in a variety of social settings. The magnetic memory board consisted of a sheet with rows showing the subject's age (0-20 years), the corresponding year, and memorable historic events that occurred during each year. As women went through the questionnaire, magnets with major life events (such as moves, school changes, births and deaths in the family) were placed in the appropriate row for the age or year in which the events occurred. All contemporary factors were asked of cases up to or around diagnosis date and of controls, up to the date of interview. The questionnaire included factors that have been summarized elsewhere $[5,12]$ as being either established or strongly suspected risk factors for breast cancer. Women who did not wish to participate in the full interview were asked to complete a brief telephone interview that included a subset of the full interview questions.

Questionnaire items pertinent to this report included detailed residential and school histories before age
21 years (such as location, ownership or rental, and dates in and out), highest level of schooling; total time of residence in Marin and other parts of the San Francisco Bay Area throughout the lifetime; ethnicity; childhood religion; parents' highest level of education and occupational characteristics at subject's age 5, 12, and 18 years; other measures of family's socioeconomic conditions including self-assessed socioeconomic status currently and before age 21 years; ages at menarche and menopause, and menopausal status; alcohol use and tobacco use and exposures; reproductive and breastfeeding history; family history of breast cancer; type and ages of use of female hormones; previous non-breast cancers; breast and cervical cancer screening practices; breast procedures; radiation treatments before breast cancer; and body weight and height at various times in life not including the times that the woman was pregnant or nursing or during the first 6 months after pregnancy.

Case interviews were conducted from December 1999 to September 2001 and control interviews were conducted from April 2000 to September 2001.

\section{Data analysis methods}

We computed frequencies of categorical variables and means, standard errors, and medians of continuous variables separately for cases and controls. Continuous data also were categorized into quartiles determined by the control group distributions. Analysis of variance was used to compare unadjusted means and Wilcoxon tests were used to compare medians of continuous variables for cases and controls. Case-control ORs and 95\% Cls were estimated for categorical and quartiled data by using logistic regression. Preliminary analyses made adjustments for 5-year age groups; these analyses included telephone interview respondents for variables asked in both the full and abbreviated interviews. After a review of results of the preliminary age-adjusted analyses, a subset of the many variables was selected for inclusion in a multivariate model; these included variables that are established or strongly suspected risk factors for breast cancer that might confound other results and those that were statistically significant in age-adjusted comparisons. Age was included in all models. In addition, ORs for residential history variables were adjusted for other variables included in the multivariate model, but not for each other, because the various residential variables were not mutually exclusive. Multivariate ORs were estimated with logistic regression and included only those subjects with in-person interviews and non-missing data for all the variables in the model.

Analyses were conducted across all age groups as well as separately for women under 50 years of age and 50 years of age and older. These two age groups were chosen for separate analyses before any data collection, because the 
direction and magnitude of several important breast cancer risk factors seem to vary by age [12]. Furthermore, we specifically chose not to analyze women separately by premenopausal and postmenopausal status, because the many perimenopausal women do not readily fit into either category. ORs of less than 1 are given two decimal places; those greater than or equal to 1 are given one decimal place to achieve comparable accuracy.

Residences at birth and before age 21 years were grouped into eight broad geographic regions based on the geographic variation of breast cancer mortality as shown in the NCl's Atlas of Cancer Mortality [15]. These regions were Northeast (Maine, New Hampshire, Vermont, Massachusetts, Connecticut, New Jersey, New York, Pennsylvania, Delaware, Washington, DC, Rhode Island, and Maryland); Great Lakes (Minnesota, lowa, Illinois, Wisconsin, Michigan, Indiana, and Ohio); Plains (North Dakota, South Dakota, Nebraska, Kansas, and Montana); South (Oklahoma, Missouri, Arkansas, Louisiana, Mississippi, Alabama, Tennessee, Kentucky, West Virginia, Virginia, North Carolina, South Carolina, Georgia, Florida, and Texas); West (Idaho, Wyoming, Utah, Colorado, Arizona, New Mexico, Alaska, and Hawaii); Northwest (Washington, Oregon, and Nevada); California; and not in the USA.

\section{Results}

\section{Subject ascertainment and interviews}

Table 1 shows participation rates for cases and controls, and the footnote gives details on the numbers and disposition of calls made through RDD. Ages and ethnicities of cases and controls that completed the full interview were very similar by study design (Table 1). Because only 15 eligible women (about 4\%) had died by the time of the study, we did not conduct proxy interviews. Of the 305 cases and 300 controls completing the in-person interview, complete data for all variables considered in the multivariate model were available for 285 cases and 286 controls.

\section{Risk factors}

Personal and family health history

As shown in Table 2, there were no substantial or statistically significant differences in the frequencies of cases and controls reporting a first-degree relative with breast cancer or a history of benign breast biopsy. Cases were somewhat more likely than controls to report previous radiation treatments for conditions other than breast cancer and the OR was significantly elevated in women under the age of 50 years (Table 2). However, on closer inspection four cases and four of the controls reported radiation treatments to the upper body, whereas two cases and no controls reported radiation treatment for plantar warts.

There were no differences in average or median age at menarche for cases and controls (average was 12.6 \pm 0.1 for cases and $12.5 \pm 0.1$ for controls) and no statistically significant trends with age at menarche in either age group (Table 2). Among women under the age of 50 years, cases were about half as likely as controls to have begun menarche at age 15 years or older, but the results were not significant (Table 2). In both age groups, cases were more likely to be premenopausal at diagnosis than controls at a similar age (Table 2). Similar proportions of cases $(6.7 \%)$ and controls $(7.3 \%)$ reported having had both ovaries removed; $2 \%$ of cases and $1 \%$ of controls had both ovaries removed before age 40 years. Cases were more likely than controls to have had a history of uterine or ovarian cancer $(\mathrm{OR}=3.0 ; 95 \% \mathrm{Cl}, 0.60-15.1)$, but the result was not statistically significant.

Some of the associations of reproductive factors differed by age group. Among women aged 50 years and older, compared with women who had never been pregnant, cases were more than twice as likely as controls to have been pregnant or parous, without breastfeeding (Table 2). However, there were no differences between these cases and controls in average age at first pregnancy (25.1 \pm 0.4 years for cases and $24.7 \pm 0.4$ years for controls) or at first birth $(26.0 \pm 0.4$ years for cases and $25.9 \pm 0.4$ years for controls) and no suggestions of trends with numbers of births (Table 2). Among women who breastfed, there was no difference between cases and controls in duration of breastfeeding (mean durations: $12.2 \pm 1.2$ months for cases, $12.8 \pm 1.3$ months for controls; categorized data presented in Table 2). Among women under the age of 50 years, cases were more likely than controls to have never been pregnant, but the differences were not statistically significant (Table 2). In categorized data, women with three or more births, or more than 12 months of breastfeeding, had significantly reduced ORs compared with women who were never pregnant (Table 2). As with women aged 50 and older, there were no trends evident for and no differences in average ages at first pregnancy (cases $25.4 \pm 0.8$ years; controls $26.3 \pm 0.7$ years) or first birth (cases $30.1 \pm 0.9$ years; controls $30.4 \pm 0.7$ years).

Significantly fewer cases than controls had ever used birth control pills in both age groups (Table 2). There were no apparent trends with total time used; compared with women who never used birth control pills, the use of birth control pills for up to 2 years, 2-6 years, $6-10$ years, and more than 10 years had ORs of 0.55 (95\% Cl, 0.33-0.93), 0.52 (95\% Cl, 0.30-0.89), 0.57 (95\% Cl, 0.32-1.0), and 0.47 (95\% Cl, 0.27-0.82), respectively. There was also no difference with current use versus past use.

About equal percentages of cases (54.7\%) and controls (55.9\%) had ever taken hormone replacement therapy and there were similar percentages who had taken combination therapy (Table 2). 


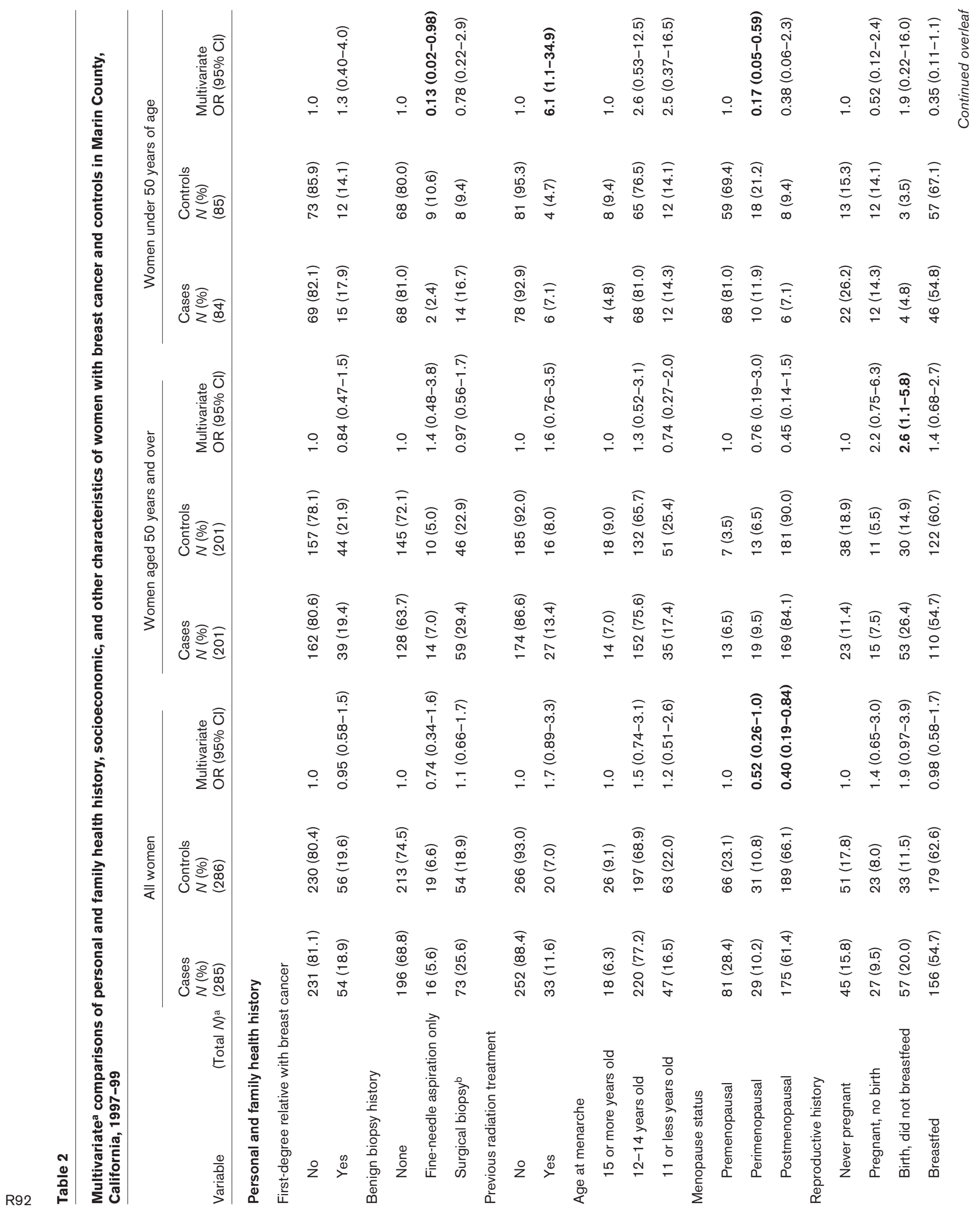




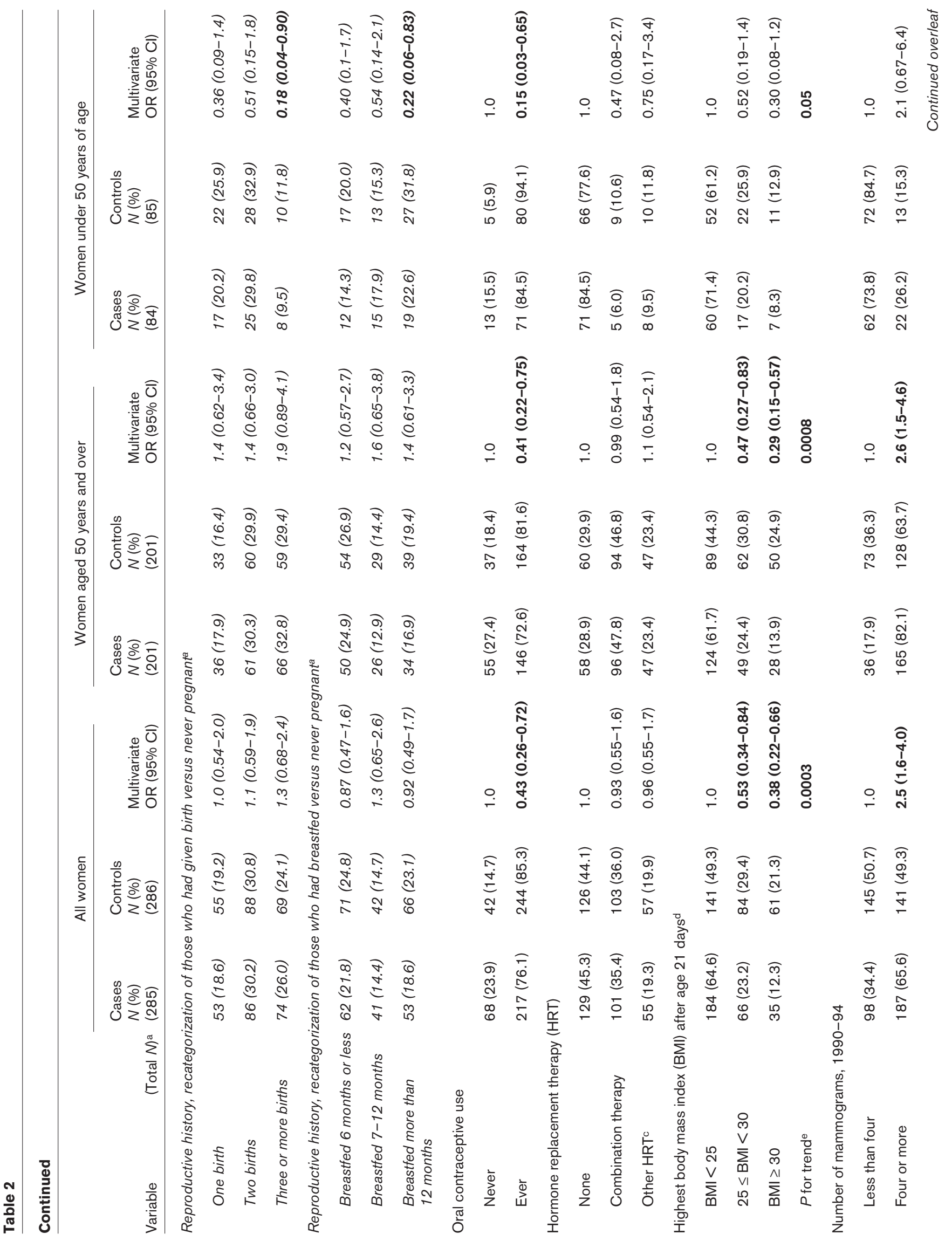




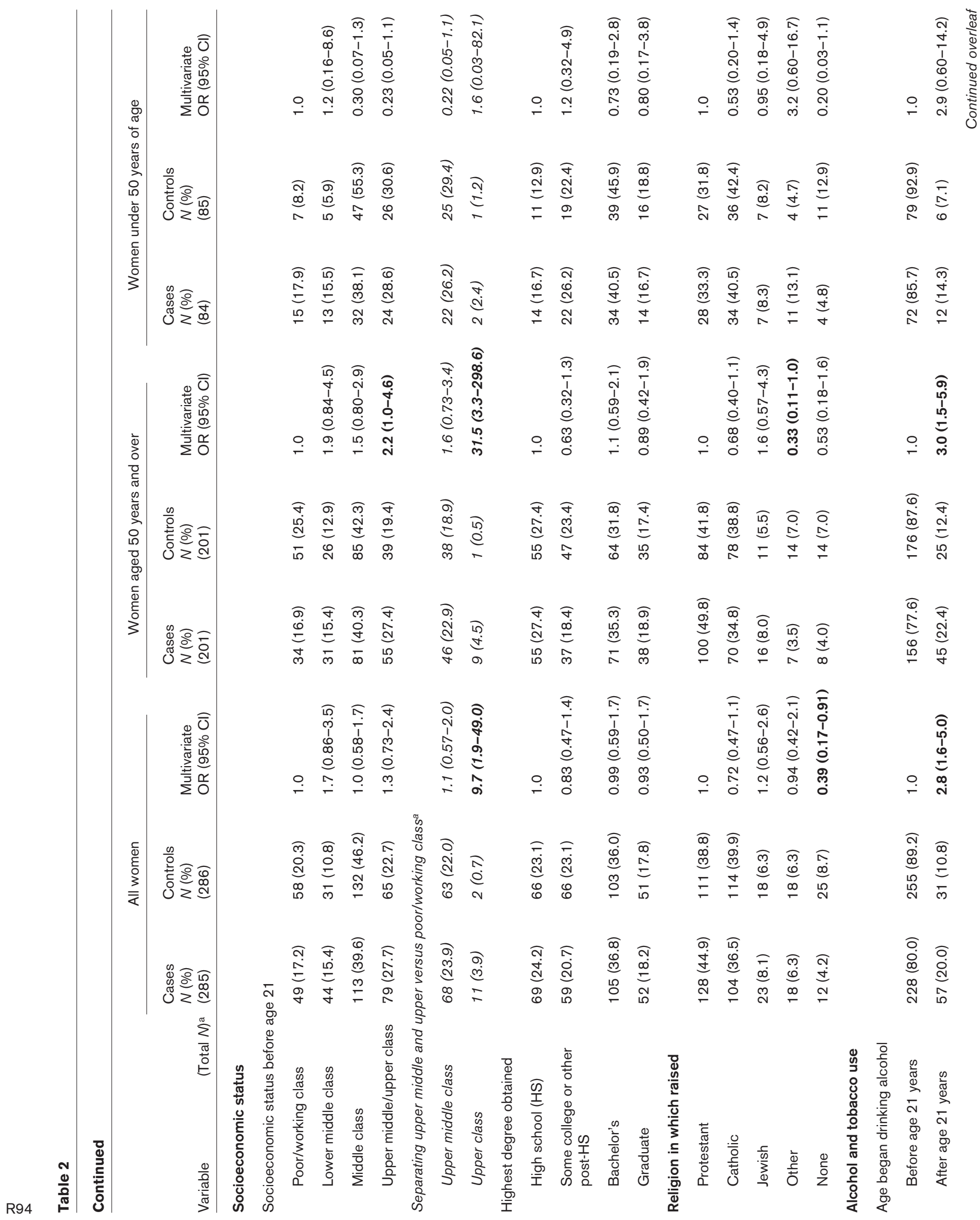




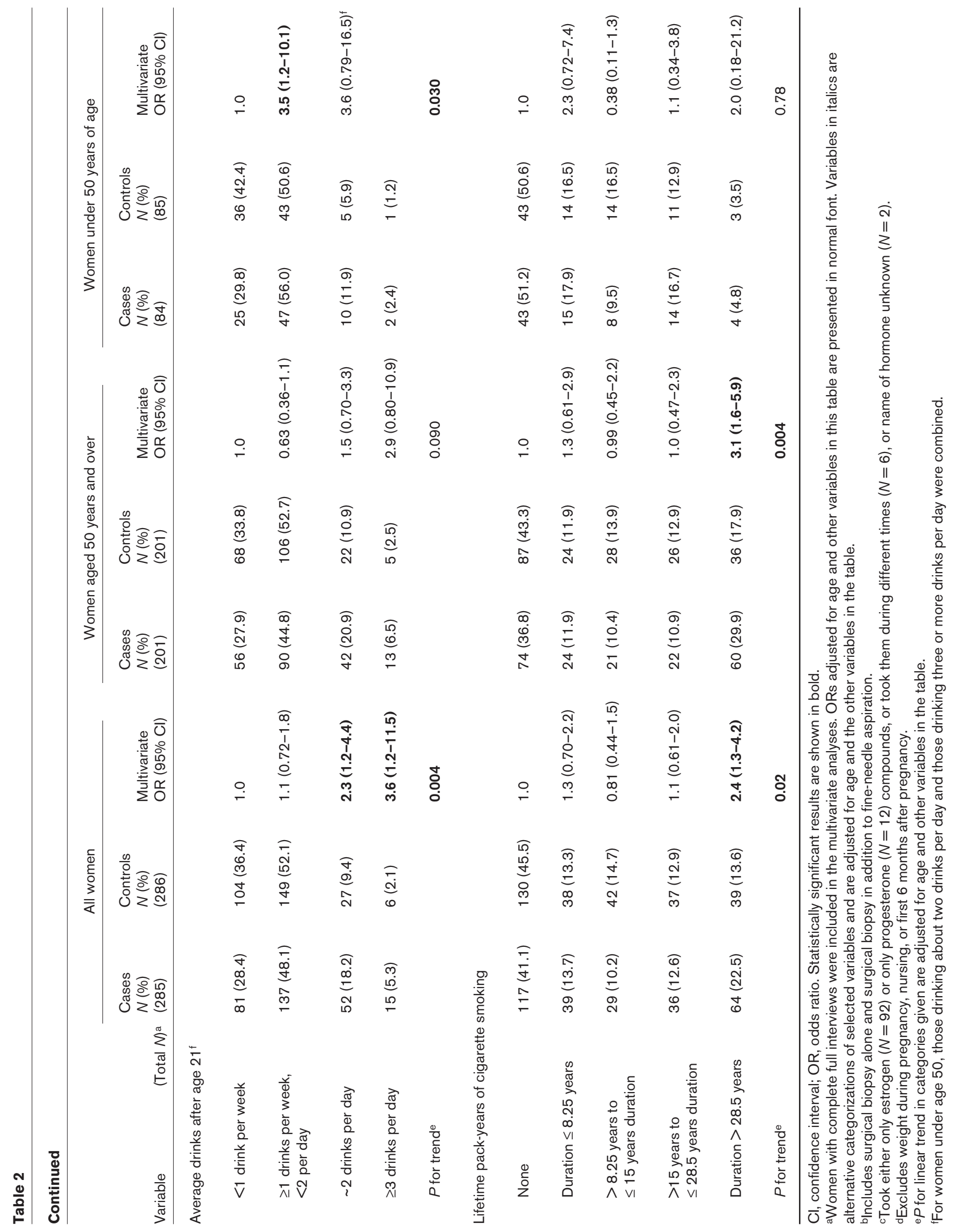


Cases and controls had similar average and median maximum lifetime heights of about 65 inches $(165 \mathrm{~cm})$. There were no notable differences in highest or lowest postpubertal body mass index before age 21 years (the median highest body mass index for both cases and controls was $20.6 \mathrm{~kg} / \mathrm{m}^{2}$, and the median lowest body mass index was $18.5 \mathrm{~kg} / \mathrm{m}^{2}$ for cases and $18.6 \mathrm{~kg} / \mathrm{m}^{2}$ for controls) or in lowest body mass index after age 21 years. However, in both age groups, the highest body mass index after age 21 years was lower in cases than in controls (the median highest body mass index was $23.9 \mathrm{~kg} / \mathrm{m}^{2}$ for cases and $25.0 \mathrm{~kg} / \mathrm{m}^{2}$ for controls); categorized data are presented in Table 2. A similar result was found by using body mass index 1 year before diagnosis for cases and before interview for controls.

We also conducted an analysis of perimenopausal and postmenopausal women that stratified body mass index by hormone replacement therapy use, adjusting for other variables in the multivariate model: in comparison with women who had not used hormone replacement therapy and had a body mass index of less than $25 \mathrm{~kg} / \mathrm{m}^{2}$, cases were significantly less likely than controls to report a highest body mass index of more than 30, regardless of whether they had used hormone replacement therapy (OR=0.36; 95\% $\mathrm{Cl}, 0.14-0.89)$ or not $(\mathrm{OR}=0.24 ; 95 \% \mathrm{Cl}, 0.08-0.75)$.

Over all and in both age groups, cases were more than twice as likely as controls to have had four or more mammograms during the period 1990-94 (Table 2). Regarding other factors related to health care access and use, all but $2 \%$ of cases and $1 \%$ of controls had health insurance. For the period 1990-94, among women under the age of 50 years, cases were somewhat less likely than controls (83\% versus $92 \%$ ) to have clinical breast exams yearly or more often, whereas cases and controls were about equally likely ( $89 \%$ of cases and $94 \%$ of controls) to have an annual or more frequent pelvic exam. During this same period, for women aged 50 years and older, about $92 \%$ of cases and $83 \%$ of controls reported annual or more frequent clinical breast exams, and $93 \%$ of cases and $87 \%$ of controls reported annual or more frequent pelvic exams and pap tests.

\section{Socioeconomic factors}

Over all ages, there were no meaningful or statistically significant differences between cases and controls for current or past socioeconomic indicators, except that cases were more likely than controls to report their family's socioeconomic strata before age 21 years as 'upper' (Table 2). However, no difference was noted when women in this small category of women $(3.9 \%$ of cases and $0.7 \%$ of controls) were combined with the 'upper middle' category (Table 2). The self-reported socioeconomic status at the time of diagnosis for cases and interview for controls
$11 \%$ of controls reported their status as 'poor', 'working class' or 'lower middle', 36\% of both cases and controls reported 'middle' and 55\% of cases and 53\% of controls reported 'upper middle' or 'upper'. Both cases and controls had a median of 16 years of education.

There was some indication that case-control associations of socioeconomic factors differed between women 50 years and older and those under 50 years. For example, among women aged 50 years and older, cases were more likely than controls to report their family socioeconomic status as 'upper or upper middle' before age 21 years (Table 2). Although this finding was largely due to the small group of women classifying themselves as 'upper', consistent with this was the observation that $33 \%$ of cases versus $22 \%$ of controls reported their fathers to have been self-employed or business owners when the subjects were 5 years old $(P<0.05)$. In contrast, among women under 50 years old there was a non-significant inverse association with increasing self-reported family socioeconomic status before age 21 years (Table 2). Consistent with this was the observation that case families spent significantly more time on average than control families in rental residences before age 21 years (6.2 \pm 0.7 years versus $4.4 \pm 0.5$ years; $P=0.03$ ) and cases were less likely than controls to report that their fathers had a college degree (40\% versus $54 \% ; P=0.05)$ or that their father held a supervisory position (for example, $50 \%$ versus $65 \%$ at subject's age 5; $P=0.05)$.

\section{Religion}

Over all ages, significantly fewer cases than controls were raised with no specific religion, and among women aged 50 and over cases were less likely than controls to report having been raised in religions other than Protestant, Catholic, or Jewish (Table 2).

\section{Alcohol consumption}

Women with breast cancer were significantly less likely than control women to report having drunk alcoholic beverages before age 21 years (Table 2). Consistent with this finding was the fact that cases also reported significantly fewer average or median numbers of drinks than controls before age 21 years (median 90 versus 120; $P=0.01$ ). However, women with breast cancer reported a higher frequency of drinking alcohol after age 21 years than controls, and there was a trend with increasing average frequency of drinking irrespective of age group (Table 2) that was statistically significant among women less than 50 years old.

\section{Tobacco exposures}

Although equivalent percentages of cases and controls had ever smoked cigarettes (59\% and 55\%, respectively), cases were more likely than controls to report more than 28.5 pack-years (Table 2; 28.5 years was the cutoff point for the upper quartile of pack-years smoked among con- 
trols). This association was also significant for women aged 50 and over, but there was no trend with increasing pack-years smoked below the highest quartile (Table 2). With regard to passive exposure to tobacco smoke, $81 \%$ of cases and $79 \%$ of controls had ever lived in a household with a smoker before age 21 years, and 60\% of cases and $62 \%$ of controls had ever lived in a household with a smoker after age 21 years, with no trend for duration of exposure in either age group.

\section{Residence history}

As shown in Table 3, there were no substantial differences in geographic area of birth or places ever lived before age 21 years among women overall or women over age 50 years. However, among women under the age of 50 years, a significantly higher percentage of cases than controls reported having been born in or having ever lived in the Northeast or Great Lakes areas before age 21 years (only geographic areas with noteworthy differences are included in the table).

Over all ages and among women aged 50 years and over, there were no notable case-control differences in the age of first living in Marin County, years before the age of 21 or lifetime years lived in Marin County or lifetime years lived in the San Francisco Bay Area (Table 3). Among women under the age of 50 years, cases had lived significantly fewer years than controls in other parts of the San Francisco Bay Area, but had similar durations of living in Marin County (Table 3).

\section{Discussion}

In this article we have focused on comparisons of generally accepted or suspected risk factors for breast cancer, including some characteristics measured both for adulthood and adolescence. Interestingly, cases and controls were strikingly similar for several factors known to influence breast cancer risk (such as family history of breast cancer, contemporary socioeconomic status, age at first birth, or use of hormone replacement therapy). For instance, although the overall rate of positive first-degree family history (19\%) among cases is similar to that reported in other recent series [16,17], the controls in this population had rates of family history comparable to those of the cases. One possible explanation for the homogeneity of cases with regard to these factors could be our focus on residents of a small, relatively homogenous, and affluent area, Marin County. The homogeneity of the Marin County population is supported by recent maps showing little variation in standardized incidence rate ratios for breast cancer among the census tracts within Marin County [18].

Another explanation could involve preferential participation in the study by controls with these recognized breast cancer risk factors over those without these factors. This seems unlikely given the high participation rates among those found to be eligible on the basis of RDD, but it is possible that there was some selection bias in women or households who were willing to answer the telephone call screening for eligibility. Further studies with alternative control ascertainment methods would be necessary to resolve this issue. In addition, appropriately adjusted comparisons of these factors in this high-risk area versus geographic areas with lower breast cancer rates would be needed to answer the question of how much of the excess of breast cancer incidence in Marin County might be due to patterns of these risk factors. Such a study is currently underway. A study that uses risk factor prevalence data from this study and other sources is also underway to estimate the attributable proportions of breast cancer due to specific risk factors.

As mentioned above, selection bias due to differential participation by cases or controls with some risk factor of interest is always a potential concern in case-control studies. In attempts to minimize this problem, we sought an abbreviated interview for those cases and controls not able or willing to participate in the full interview. The preliminary age-adjusted analyses of individual risk factors used all women for whom information was available. There were not any notable differences in magnitude of the age-adjusted ORs that used all 657 participants and the multivariate results based on 571 subjects with complete information.

Despite homogeneity in current socioeconomic status, our study suggests that among women aged 50 years and older, breast cancer cases might have been more likely than controls to have grown up in more affluent families, although the results are largely due to the small proportion of subjects reporting their family's socioeconomic status as 'upper' before age 21 years. In contrast, for women under the age of 50 years, breast cancer cases were somewhat less likely than controls to have grown up in more affluent families. That an effect of pre-adult socioeconomic status existed in the women aged 50 and over that was independent of contemporary socioeconomic status as well as established reproductive, menstrual, and other factors such as body size and alcohol consumption suggests that further attention is needed to understand the relationship of childhood or adolescent socioeconomic factors and breast cancer.

Almost all women in this study had health insurance, and whereas women with breast cancer were more likely than controls to report having had four or more mammograms in the period 1990-94, they were about equally likely to report obtaining pelvic exams over the same period. This suggests that women who subsequently developed breast cancer might have had more mammograms than controls because they had more breast problems rather than because they were, in general, more regular users of cancer screening services than controls. 


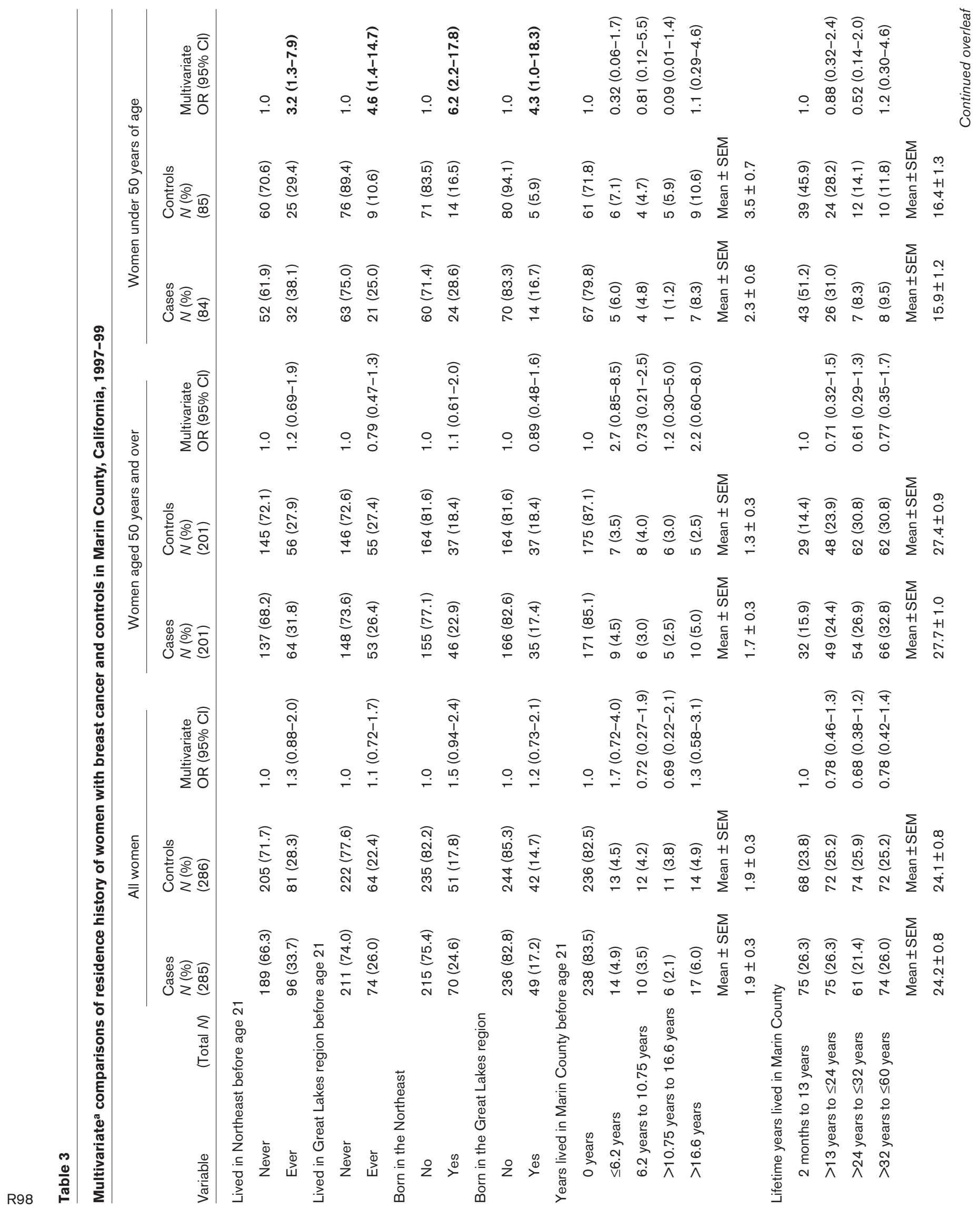




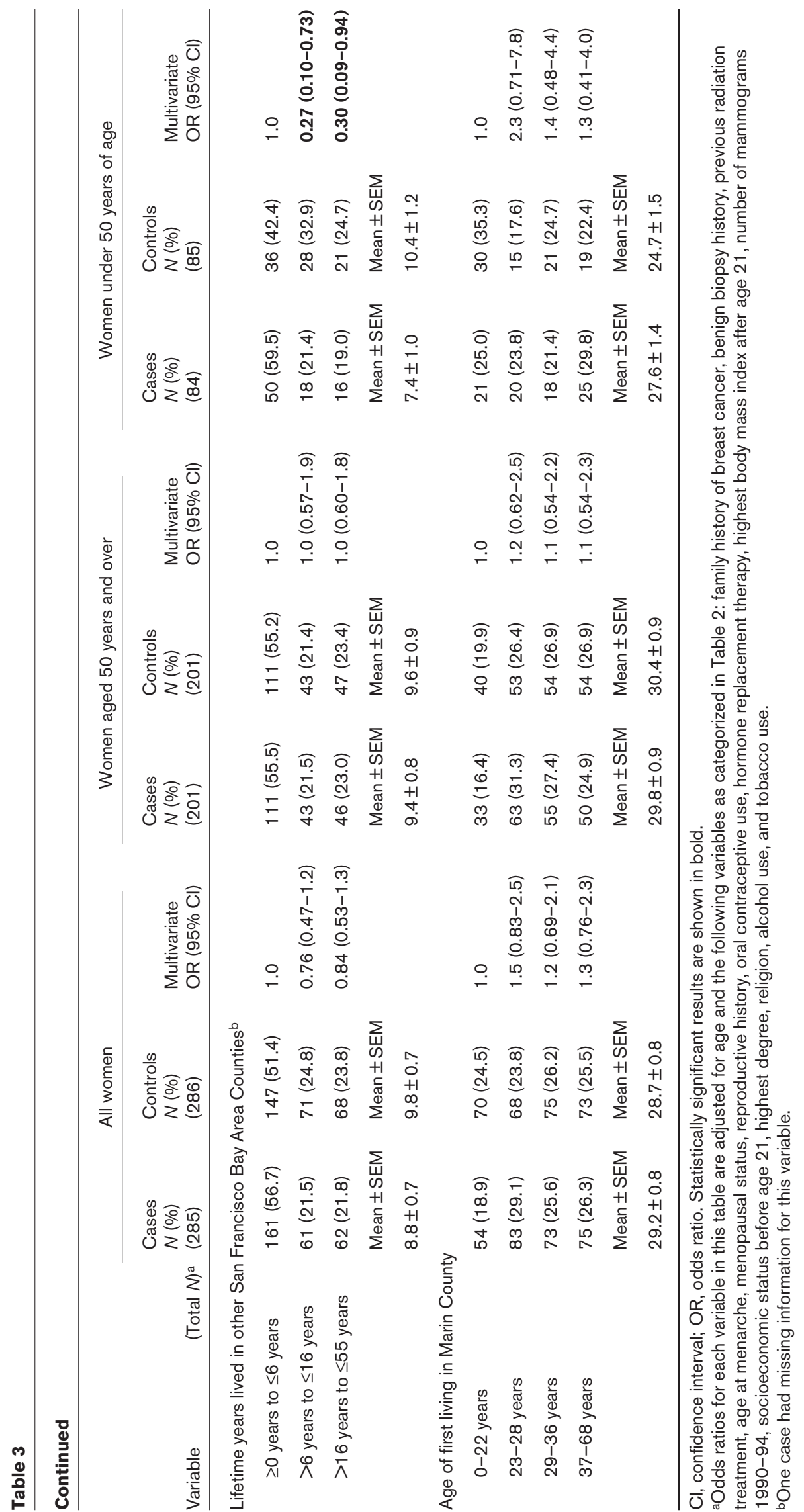


Interestingly, in both age groups, women who had given birth but had not breastfed had a higher risk for breast cancer than women who had never been pregnant, women who had been pregnant but not parous, and women who had been parous and breastfed, although the result was statistically significant only in those aged 50 years and older. In addition, among women under the age of 50 years, women who breastfed for 12 months or more had significantly reduced odds of breast cancer. This finding is consistent with a large collaborative study [19] showing breastfeeding to be protective for breast cancer through hormonal or other mechanisms. That report suggested an urgent need to fully understand the mechanisms of the protective effect of breastfeeding so that some intervention mimicking breastfeeding could be developed and offered to women who have not breastfed.

It is also possible that inability to lactate or suppressing lactation after giving birth might have a deleterious effect on breast physiology [20], but we did not request this information from subjects. Given the relatively late ages of first birth in this population, one might not expect to see a protective effect of having ever given birth, because the well-established protective effects of pregnancy seem to be reversed for first births at about age 30 years or later $[21,22]$. This study had a higher percentage of nulliparous women ( $25 \%$ of cases and $26 \%$ of controls) than a recent study conducted in the Seattle area $(13 \%$ of cases and 14\% of controls) [17]. However, the California Teachers Study cohort, with a similarly elevated rate of invasive breast cancer of 151 per 100,000 person years among non-Hispanic white women between 1995 and 1998 , reported a similar percentage of nulliparous women (27\%) among participants without breast cancer in 1995 [23].

Consistent with previous findings [12] was our observation that cases were more likely to be premenopausal than controls, suggesting a later age at menopause. Despite the absence of a strong difference in age at menarche between cases and controls in this group, those women who developed breast cancer had relatively later menopause, indicating a greater number of years of active menstruation. Starting menarche at age 11 years or earlier is considered an established breast cancer risk factor, and starting menarche at age 15 years or older is considered an established protective factor [12]. In the present study, cases were somewhat less likely than controls to start menarche at age 15 years or older, but cases were not more likely than controls to have early menarche. A similar lack of association of age at menarche with breast cancer status was apparent in the large recent study by Marchbanks and colleagues [16]. The distribution of age at menarche reported by control women in Marin County was similar to that observed for women in the California
Disagreement remains in the literature on the direction and magnitude of effect, if any, of oral contraceptives on breast cancer risk $[12,16,24]$. Despite large studies designed to address such differences, chance, selection factors, changes in formulations, patterns of use, and different background risk for breast cancer might account for some of the variation in findings. In this Marin County study, we found a rather strong and significant inverse association of oral contraceptive use and breast cancer. However, the lack of case-control differences by duration or latency of use argues against a causal connection and suggests that oral contraceptive use might be a surrogate for some other protective behavior or exposure. Given the very high rate of use of oral contraceptives in this population, an alternative explanation could be that factors or conditions contraindicating the use of oral contraceptives might increase the risk for breast cancer. In future studies it might be worthwhile to assess why women chose to use, or not to use, oral contraceptives.

In addition, there was no indication of large differences in hormone replacement therapy use among cases and controls, which was similar to a recent Seattle case-control study [17], although any use of hormone replacement therapy was somewhat higher in the Marin population $(55-56 \%)$ than in the women in the Seattle study $(49-51 \%)$. In addition, the specificity of questions about types of hormone replacement therapy used and the sample size of this Marin study is insufficient to rule out the small increased risk (25-30\%) observed in larger studies [25,26] or to examine in detail different hormone replacement therapy regimens, duration, or age of use. Appropriately adjusted comparisons of rates of hormone replacement therapy use in this area and geographic areas with lower breast cancer rates would be informative regarding the relationship of hormone replacement therapy use to the elevated breast cancer incidence in Marin County.

Many studies have examined breast cancer in relation to body weight, height, and overall body size (as measured by body mass index). The general consensus has been that increased height might be associated with increased risk for breast cancer, and that obesity might increase risk in women aged 50 years or over or who are postmenopausal but might decrease risk in women under the age of 50 years [27-30]. Highest adult body mass index was strongly inversely related to breast cancer status both in women under the age of 50 years and, unexpectedly, in those aged 50 years and older. The average highest body mass indexes reported in this study (24.9 and $26.5 \mathrm{~kg} / \mathrm{m}^{2}$ for cases and controls, respectively) were in the same range reported for subjects' body mass index 5 years previously in a recent large US study (25.5 and $25.8 \mathrm{~kg} / \mathrm{m}^{2}$ for cases and controls, respectively) [16] and participants in the California Teachers Study in 1995 $\left(24.8 \mathrm{~kg} / \mathrm{m}^{2}\right)$ [23]. 
One explanation for the finding could be that mechanisms of weight maintenance might have contributed to the association. Because we did not ask about adult or recent physical activity in the interview, we cannot assess the impact of these factors on the body size results. In the Nurses' Health Study cohort, Huang and colleagues [31] reported that among postmenopausal women, weight gain was consistently associated with an increased risk for breast cancer only among women who had not used hormone replacement therapy. We found that cases had lower highest body mass index than controls both among women who had used hormone replacement therapy and among women who had not. It is possible in this affluent community that perimenopausal and postmenopausal women who are not taking prescribed hormone replacement therapy could be eating foods or taking herbal or other non-prescribed supplements to ameliorate menopausal symptoms that might simulate the effects of hormone replacement therapy. Unfortunately we did not collect the data to test this possibility, but such questions should be included in future studies.

Alcohol use has been increasingly associated with both premenopausal and postmenopausal breast cancer risk $[32,33]$, including a report from the California Teachers Study, showing that recent alcohol consumption increases risk for breast cancer [34]. We found that although women with breast cancer were less likely than controls to have begun drinking alcohol before age 21 years, they were much more likely than controls to consume two or more drinks per day on average as adults. That the ORs for alcohol consumption in this study were generally higher than those reported in other studies suggests a possible interaction of alcohol consumption with other factors that have a high prevalence in this population.

Cigarette smoking has not been consistently associated with breast cancer risk [12]. The elevated OR observed in this study for the highest quartile of pack-years smoked might be due to differential participation by controls with a lower pack-year history or differential reporting of smoking history by cases and controls. It is also possible that smoking history was confounded by factors not measured in this study, such as physical activity.

For women under the age of 50 years, women with breast cancer were much more likely than controls to have been born or lived before age 21 years in the Northeastern or Great Lakes regions of the USA, regions that were in the upper deciles of breast cancer mortality both for the period 1950-69 and for the period 1970-94 [15]. Geocoding and other refinements of residence information might help to clarify which areas of first or early life residences might be associated with later risk for breast cancer.

The similar average ages of first residence and total years lived in Marin for cases and controls do not support (but cannot completely rule out) the hypothesis that there is some agent specific to Marin County, such as a particular water source, that might increase the risk for breast cancer. These findings do not imply that environmental factors are unimportant in breast cancer causation. On the contrary, our and others' findings of increased risk with frequency of alcohol consumption show that a woman's personal environment can strongly influence her breast cancer risk. In addition, our result of higher risk among women under the age of 50 years who lived in the Northeast or Great Lakes regions before age 21 years might suggest a role of early environmental factors.

Because control women in this study had such high rates of health insurance and use of basic screening services, it seems likely that they truly did not have breast cancer at the time of interview. This was despite a higher proportion of controls with established breast cancer risk factors such as family history and nulliparity than observed in control subject groups in other recent studies. Given this observation, a further intensive study of this or other similarly high-incidence populations might reveal factors proximate to breast cancer diagnosis that could enrich our understanding of breast cancer etiology in a high-incidence population. Some provocative clues from this first analysis suggest that some as yet unidentified correlate of oral contraceptive use might be protective and that consuming two or more alcoholic drinks per day might be deleterious for women in this high-incidence population. We were unable to examine the effects of other provocative exposures previously proposed by others, including exposures to light at night [35] and polycyclic aromatic hydrocarbons [36]. The observed early socioeconomic differences combined with a lack of differences in age at menarche or early body size also suggest that attention might be turned to patterns of early infection (or lack of infection) and immune response [37].

This study is among the first reported in which community advocates took part in all aspects of the research process, from formulating questions of interest and questionnaire design to monitoring progress of subject recruitment and data analysis and interpretation at regular meetings. This study provides important information for planning additional studies to understand reasons for breast cancer in this or other high-risk geographic areas characterized by a socioeconomically affluent population.

\section{Conclusions}

Women with breast cancer and controls had very similar distributions of age of first living and years lived in Marin County, suggesting that the County's high breast cancer rates are probably not due to geographically specific exposures. Despite similar distributions for several known breast cancer risk factors, case-control differences in alcohol consumption suggest that breast cancer risk in 
this population might be modifiable. This study might be useful to communities and researchers concerned with understanding the basis of, and means of mitigating, high local or regional rates of breast cancer. Intensive study of high-incidence areas might reveal other important risk factors proximate to diagnosis.

\section{Competing interests}

None declared.

\section{Acknowledgements}

We thank Ruth Fleshman and Mary Mizroch for interviewing, and Nancy Lynn, Natalie Carter, Colin Leary, Nancy Brennan, Chantal Jaycox, and Joe Klimmeck for study assistance. We are also grateful for the time and commitment of the study's participants. This work was supported by grants 4AB-14801 and 5BB-1201 from the California Breast Cancer Research Program's Community Research Collaboration funding mechanism.

\section{References}

1. West D, Glaser S, Prehn A: Status of Breast Cancer Research in the San Francisco Bay Area. Union City: Northern California Cancer Center; 1998.

2. Clarke CA, Glaser SL, West DW, Ereman RR, Erdmann CA, Barlow JM, Wrensch M: Breast Cancer incidence and mortality trends in an affluent population: Marin County, California, USA, 1990-1999. Breast Cancer Res 2002, 4:R13.

3. Ziegler RG, Hoover RN, Pike MC, Hildesheim A, Nomura AM, West DW, Wu-Williams AH, Kolonel LN, Horn-Ross PL, Rosenthal JF: Migration patterns and breast cancer risk in AsianAmerican women. J Natl Cancer Inst 1993, 85:1819-1827.

4. Kelsey JL, Horn-Ross PL: Breast cancer: magnitude of the problem and descriptive epidemiology. Epidemiol Rev 1993, 15:7-16.

5. Kelsey JL: Breast cancer epidemiology: summary and future directions. Epidemiol Rev 1993, 15:256-263.

6. Henderson BE, Pike MC, Bernstein L, Ross RK: Breast cancer. In: Cancer Epidemiology and Prevention. Edited by Schottenfeld D, Fraumeni JF. New York: Oxford University Press; 1996:1022-1039.

7. Robbins AS, Brescianini S, Kelsey JL: Regional differences in known risk factors and the higher incidence of breast cancer in San Francisco. J Natl Cancer Inst 1997, 89:960-965.

8. Sturgeon SR, Schairer C, Gail M, McAdams M, Brinton LA, Hoover RN: Geographic variation in mortality from breast cancer among white women in the United States. J Natl Cancer Inst 1995, 87:1846-1853.

9. Maskarinec G, Wilkens L, Meng L: Mammography screening and the increase in breast cancer incidence in Hawaii. Cancer Epidemiol Biomarkers Prev 1997, 6:201-208.

10. Prehn AW, West DW: Evaluating local differences in breast cancer incidence rates: a census-based methodology (United States). Cancer Causes Control 1998, 9:511-517.

11. NCCC: Breast cancer incidence in Marin County: relationship to known risk factors. Northern California Cancer Center Greater Bay Area Cancer Registry Report 1997, 7:1-6.

12. Kelsey JL, Bernstein L: Epidemiology and prevention of breast cancer. Annu Rev Public Health 1996, 17:47-67.

13. Waksberg J: Sampling methods for random digit dialing. J Am Statist Assoc 1978, 73:40-46.

14. Harlow BL, Davis S: Two one-step methods for household screening and interviewing using random digit dialing. $A m J$ Epidemiol 1988, 127:857-863.

15. Devesa SS, Grauman DJ, Blot WJ, Pennello GA, Hoover RN, Fraumeni JF: Atlas of cancer mortality in the United States, 195094. Bethesda, Maryland: National Institute of Health, National Cancer Institute; 1999.

16. Marchbanks PA, McDonald JA, Wilson HG, Folger SG, Mandel MG, Daling JR, Bernstein L, Malone KE, Ursin G, Strom BL, Norman SA, Wingo PA, Burkman RT, Berlin JA, Simon MS, Spirtas R, Weiss LK: Oral contraceptives and the risk of breast cancer. N Engl J Med 2002, 346:2025-2032.

17. Davis S, Mirick DK, Stevens RG: Residential magnetic fields and the risk of breast cancer. Am J Epidemiol 2002, 155:446-454.
18. Marin County Department of Health and Human Services: http://199.88.77.93/depts/HH/main/epi/map.cfm

19. Collaborative Group on Hormonal Factors in Breast Cancer: Breast cancer and breastfeeding: collaborative reanalysis of individual data from 47 epidemiological studies in 30 countries, including 50302 women with breast cancer and 96973 women without the disease. Lancet 2002, 360:187-195.

20. Lipworth L, Bailey LR, Trichopoulos D: History of breast-feeding in relation to breast cancer risk: a review of the epidemiologic literature. J Natl Cancer Inst 2000, 92:302-312.

21. Kelsey JL, Gammon MD, John EM: Reproductive factors and breast cancer. Epidemiol Rev 1993, 15:36-47.

22. Pathak DR, Osuch JR, He J: Breast carcinoma etiology: current knowledge and new insights into the effects of reproductive and hormonal risk factors in black and white populations. Cancer 2000, 88:1230-1238.

23. Bernstein L, Allen $M$, Anton-Culver $H$, Deapen $D$, Horn-Ross $P L$, Peel D, Pinder R, Reynolds P, Sullivan-Halley J, West DW, Wright W, Ziogas A, Ross RK: High breast cancer incidence rates among California teachers: results from the California Teachers Study (United States). Cancer Causes Control 2002, 13:625-635.

24. Collaborative Group on Hormonal Factors in Breast Cancer: Breast cancer and hormonal contraceptives: further results. Contraception 1996, 54:1S-106S.

25. Collaborative Group on Hormonal Factors in Breast Cancer: Breast cancer and hormone replacement therapy: collaborative reanalysis of data from 51 epidemiological studies of 52,705 women with breast cancer and 108,411 women without breast cancer. Lancet 1997, 350:1047-1059.

26. Writing Group for the Women's Health Initiative Investigators: Risks and benefits of estrogen plus progestin in healthy postmenopausal women: principal results from the Women's Health Initiative randomized controlled trial. JAMA 2002, 288:321-333.

27. Hunter DJ, Willett WC: Diet, body size, and breast cancer. Epidemiol Rev 1993, 15:110-132.

28. Brinton LA, Swanson CA: Height and weight at various ages and risk of breast cancer. Ann Epidemiol 1992, 2:597-609.

29. Stoll BA: Teenage obesity in relation to breast cancer risk. Int $J$ Obes Relat Metab Disord 1998, 22:1035-1040.

30. Cleary MP, Maihle NJ: The role of body mass index in the relative risk of developing premenopausal versus postmenopausal breast cancer. Proc Soc Exp Biol Med 1997, 216:28-43.

31. Huang $Z$, Hankinson SE, Colditz GA, Stampfer MJ, Hunter DJ, Manson JE, Hennekens CH, Rosner B, Speizer FE, Willett WC: Dual effects of weight and weight gain on breast cancer risk. JAMA 1997, 278:1407-1411.

32. Bowlin SJ, Leske MC, Varma A, Nasca P, Weinstein A, Caplan L: Breast cancer risk and alcohol consumption: results from a large case-control study. Int J Epidemiol 1997, 26:915-923.

33. Singletary KW: Alcohol and breast cancer: interactions between alcohol and other risk factors. Alcohol Clin Exp Res 1996, 20:57A-61A.

34. Horn-Ross PL, Hoggatt KJ, West DW, Krone MR, Stewart SL, Anton H, Bernstei CL, Deapen D, Peel D, Pinder R, Reynolds P, Ross RK, Wright W, Ziogas A: Recent diet and breast cancer risk: the California Teachers Study (USA). Cancer Causes Control 2002, 13:407-415.

35. Davis S, Mirick DK, Stevens RG: Night shift work, light at night, and risk of breast cancer. J Natl Cancer Inst 2001, 93:1557-1562.

36. Gammon MD, Santella RM, Neugut Al, Eng SM, Teitelbaum SL, Paykin A, Levin B, Terry MB, Young TL, Wang LW, Wang Q, Britton JA, Wolff MS, Stellman SD, Hatch M, Kabat GC, Senie R, Garbowski G, Maffeo C, Montalvan P, Berkowitz G, Kemeny M, Citron M, Schnabel F, Schuss A, Hajdu S, Vinceguerra V: Environmental toxins and breast cancer on Long Island. I. Polycyclic aromatic hydrocarbon DNA adducts. Cancer Epidemiol Biomarkers Prev 2002, 11:677-685.

37. Richardson A: Is breast cancer caused by late exposure to a common virus? Med Hypotheses 1997, 48:491-497.

\section{Correspondence}

Margaret Wrensch PhD, Department of Epidemiology and Biostatistics, Box 1215, University of California, San Francisco, 44 Page Street Suite 503, University of California, San Francisco, San Francisco, CA 94102, USA. Fax: +1 415502 1787; e-mail: wrensch@itsa.ucsf.edu 